\title{
Positive effect of joint activation of mTOR-dependent and mTOR-independent pathways of autophagy in the treatment of two experimental models of neurodegeneration
}

\author{
Alexander B. Pupyshev \\ Scientific Research Institute of \\ Physiology and Basic Medicine \\ Novosibirsk, Russia \\ pupyshevab@physiol.ru \\ Anna A. Akopyan \\ Scientific Research Institute of \\ Physiology and Basic Medicine \\ Novosibirsk, Russia \\ annaaleksanovna@mail.ru \\ Mikhail V. Tenditnik Scientific \\ Research Institute of Physiology and \\ Basic Medicine Novosibirsk, Russia \\ m.v.tenditnik@physiol.ru
}

\author{
Nina I. Dubrovina \\ Scientific Research Institute of \\ Physiology and Basic Medicine \\ Novosibirsk, Russia \\ dubrov@physiol.ru \\ Marina V. Ovsyukova \\ Scientific Research Institute of \\ Physiology and Basic Medicine \\ Novosibirsk, Russia maryov@ngs.ru \\ Tatiana A. Korolenko \\ Scientific Research Institute of \\ Physiology and Basic Medicine \\ Novosibirsk, Russia \\ t.a.korolenko@physiol.ru
}

\author{
Maria A. Tikhonova \\ Scientific Research Institute of \\ Physiology and Basic Medicine \\ Novosibirsk, Russia \\ tikhonovama@physiol.ru
}

\begin{abstract}
Autophagy is a part of the cellular protein quality control system and is able to suppress neurodegeneration via weakening pathogenic proteins accumulation. Meanwhile possibilities of different ways of autophagy induction in treatment of experimental neurodegeneration are poorly known. Here we used pharmacological models of Alzheimer's disease (AD) and Parkinson's disease (PD) induced correspondingly by amyloid-beta and MPTP administration in mice. mTORdependent autophagy was induced by rapamycin and mTORindependent autophagy was activated by trehalose. Both drugs caused increase in autophagy (according to LC3-II level) in the affected brain structures, namely, the striatum and s. nigra in PD model and the hippocampus in AD model. Neuronal loss was reduced according to the expression of tyrosine hydroxylase (PD) or neuronal density (AD). Neuroinflammation was sharply reduced according to IBA1 microglial marker in the hippocampus (AD). Trehalose was more active than rapamycin, but the highest effect was achieved by their combined application. An obvious restoration of cognitive function in the passive avoidance test for memory and learning using all three treatment approaches has been found for both models of neurodegeneration. Recovery of emotional activity, as measured by a decrease in anxiety in AD model, was significant only for the combination of the drugs. Cytoprotective and therapeutic effects of the drugs in AD model were eliminated by blocking autophagy with 3methyladenine. Thus, a more noticeable neuroprotective effect is produced by trehalose in comparison with rapamycin while a joint activation of the mTOR-dependent and mTORindependent autophagy pathways, by rapamycin and trehalose, respectively, renders the highest impact.
\end{abstract}

Keywords - animal models, Alzheimer's disease, Parkinson's disease, neuroprotection, autophagy, mTORdependent, mTOR-independent, rapamycin, trehalose, LC3-II, IBA1, passive avoidance test.

Motivation and Aim

Motivation

Autophagy is a part of cellular protein quality control system and renders cytoprotective effect in aberrant misfolded protein accumulation occurring in neurodegeneration processes. Canonical mTOR-dependent pathway of autophagy activation induced by rapamycin is connected with some side effects. mTOR-independent stimulation of autophagy started up by trehalose seemingly is devoided of side impacts and could be a better agonist. The positive effects of the drugs in neurodegeneration are described mostly in in vitro models [1].

Aim

Here we tried to estimate neuroprotective effects of rapamycin and trehalose in their separate and joint application in two animal models of neurodegeneration, i.e. Alzheimer's and Parkinson's diseases.

\section{Methods}

We used pharmacological models of Alzheimer's disease (AD) and Parkinson's disease (PD). The first was induced by intraventricular injection of amyloid- $\beta$ 25-35 fragment, and the second was reproduced by systemic MPTP administration into mice C57Bl/6 [2]. Rapamycin was applied i/p in dose $10 \mathrm{mg} / \mathrm{kg}$ body weight, trehalose was included in drinking $(2 \%)$. Their impacts were evaluated by means of immunohistochemistry of autophagy marker LC3II, level of amyloid- $\beta$, tyrosine hydroxylase, neuronal density (Nissl staining), behavioral tests ("memory of fear", T-maze, "open field").

\section{Results}

Both drugs caused increase in autophagy (according to LC3-II level) in the affected brain structures, namely, the striatum and s. nigra in PD model and the hippocampus in AD model. Neuronal loss was reduced according to the expression of tyrosine hydroxylase (PD) or neuronal density (AD). Neuroinflammation was sharply reduced according to IBA1 microglial marker in the hippocampus (AD). Trehalose was predominantly more active than rapamycin, but the highest effect was achieved by their combined application. An obvious restoration of cognitive function in the passive avoidance test for memory and learning using all three treatments has been found for both models of neurodegeneration. Recovery of emotional activity, as measured by anxiety in $\mathrm{AD}$ model, was significant only for 
the combination of the drugs. Cytoprotective and therapeutic effects of the drugs in $\mathrm{AD}$ model were eliminated by blocking autophagy with 3-methyladenine. Thus, a more noticeable neuroprotective effect is produced by trehalose in comparison with rapamycin while a joint activation of the mTOR-dependent and mTOR-independent autophagy pathways, by rapamycin and trehalose, respectively, has the highest impact.

\section{ACKNOWLEDGMENT}

The study was supported by budgetary funding for basic scientific research of the Scientific Research Institute of Physiology and Basic Medicine (theme No. AAAA-A16-
116021010228-0) and the Russian Foundation for Basic Research (grant No. 16-04-01423-a).

\section{REFERENCES}

[1] Sarkar S. et al., (2007). Trehalose, a novel mTOR-independent autophagy enhancer, accelerates the clearance of mutant Huntingtin and alpha-synuclein. J. Biol. Chem. 282 (8): 5641-5652.

[2] Pupyshev A.B. et al., (2019). Therapeutic activation of autophagy by combined treatment with rapamycin and trehalose in a mouse MPTP induced model of Parkinson's disease. Pharmacol. Biochem. Behav.177(1): 1-11. 